\title{
Sublocalization, Superlocalization, and Violation of Standard Single Parameter Scaling in the Anderson Model
}

\author{
Jan W. Kantelhardt ${ }^{1,2}$ and Armin Bunde ${ }^{1}$ \\ ${ }^{1}$ Institut für Theoretische Physik III, Justus-Liebig-Universität Giessen, D-35392 Giessen, Germany \\ ${ }^{2}$ Center for Polymer Studies and Department of Physics, Boston University, Boston, MA 02215, USA \\ (submitted: January 19, 2002, revised version: April 24, 2002)
}

\begin{abstract}
We discuss the localization behavior of localized electronic wave functions in the one- and two-dimensional tight-binding Anderson model with diagonal disorder. We find that the distributions of the local wave function amplitudes at fixed distances from the localization center are well approximated by log-normal fits which become exact at large distances. These fits are consistent with the standard single parameter scaling theory for the Anderson model in 1d, but they suggest that a second parameter is required to describe the scaling behavior of the amplitude fluctuations in $2 \mathrm{~d}$. From the log-normal distributions we calculate analytically the decay of the mean wave functions. For short distances from the localization center we find stretched exponential localization ("sublocalization") in both, $d=1$ and $d=2$. In $d=1$, for large distances, the mean wave functions depend on the number of configurations $N$ used in the averaging procedure and decay faster that exponentially ("superlocalization") converging to simple exponential behavior only in the asymptotic limit. In $d=2$, in contrast, the localization length increases logarithmically with the distance from the localization center and sublocalization occurs also in the second regime. The $N$-dependence of the mean wave functions is weak. The analytical result agrees remarkably well with the numerical calculations.
\end{abstract}

PACS numbers: 71.23.An, 73.20.Fz

\section{INTRODUCTION}

The Anderson model is the standard model for the electronif properties of disordered solids, 1 for reviews, see e. $g$. Refs 2 t 4 The wave functions are known to be localized in dimensions $d \leq 2$ (except at the band center for non-diagonal disorder) as well as for sufficiently strong disorder in $d>2$. The shape of these localized wave functions is believed to be characterized by an asymptotically exponential decay, which can be described most effectively $\gamma$ or its inverse, the localization length $\lambda$. The simple exponential shape has been the basis of many calculations and models, e. g. Mott's hopping conductivity and tunneling on quantum dots. Even for other models of disordered matter, e. g. for percolation models, 1 simple exponential localization of the wave functions has been assumed based on the analogy with the Anderson model.

In addition to the exponential decay at large distances from the localization center, the wave functions in the Apderson model exhibit large fluctuations of their amplitudes. 3 These fluctuations are rather important for transport properties, e. g. for the distribution of mesoscopic conductances, since the core region of the wave functions is dominated by the fluctuations, and the exponential decay can only be observed at distances much larger than the localization length $\lambda$ for sin gle wave functions. In the single-parameter scaling theory (SPST) it has been assumed that the whole distribution of the conductances or Lyapunov exponents for an ensemble of configurations can be described by one parameter only, e. g. the width of the distribution of values of Lyapunov exponents depends on the average Lyapunov exponent $\gamma$ in a universal way. Hence, the localization length $\lambda=1 / \gamma$ could be used as single relevant scaling parameter fully characterizing the localization behavior.
Very recently, there has been a growing interest in the question, if the behavior of the fluctuations of the wave fynctions are universal and can be described within the SPST, 10 see also Refs.11 11 the interest is partly motivated by the experimental observations of a transition in the behavior of the conductance of low density two dimensional semiconductor devices from an insulating like temperature dependence at low densities to a metallic one at higher densities. ${ }^{16}$ This apparent localization-delocalization transition in $d=2(2 \mathrm{~d})$ is at odd with the SPST for non-interacting electrons.

In this paper, we investigate the distribution of the amplitudes of Anderson localized wave function in $d=1$ (1d) and $2 \mathrm{~d}$ at given distances $r$ from the localization center, which allows a test of the SPST hypothesis. We show that the SPST hypothesis holds perfectly in $1 \mathrm{~d}$, while deviations from the standard SPST occur for localized wave functions for the Anderson model in 2d. Deviations also occur for the percolation model in $2 \mathrm{~d}$ and $3 \mathrm{~d}$. In both cases, the width of the amplitude distribution is characterized by a second parameter that depends on the type of disorder. In addition, we find a clear logarithmic system-size dependence of the localization length $\lambda$ for the Anderson model in 2d, but no significant dependence on the boundary conditions.

From the amplitude distributions we can calculate analytically the behavior of the mean wave functions at sufficiently large distances from the localization center. We find that simple exponential decay as usually anticipated occurs only in $1 \mathrm{~d}$ in the asymptotic limit where the wave function has decayed to extremely small values. At finite distances from the localization center, we observe two different localization regimes. In the first regime, at short distances from the localization center, we find stretched exponential localization with a localization exponent below one ("sublocalization") in $1 \mathrm{~d}$ and $2 \mathrm{~d}$. In the second regime, at larger distances, the averages in $1 \mathrm{~d}$ de- 
pend on the number of configurations and we observe "superlocalization", where the localization exponent is greater than one. The crossover from the sublocalization to the superlocalization regime increases logarithmically with the number of configurations. In the asymptotic limit, the localization exponent converges to one, yielding the theoretically predicted simple exponential behavior. In $d=2$, however, we observe the surprising result that the sublocalization of the mean wave functions remains also in the second regime.

The paper is organized as follows: In Section II we briefly introduce the Anderson model and summarize some of the known results. In Section III we discuss distributions of wave function amplitudes, beginning with a review of the predictions of the standard SPST and the random matrix theory. Then we introduce an amplitude statistics which is most useful for the characterization of localized wave functions, and present our numerical results for this amplitude statistics. In Section IV we calculate analytically the decay of the mean wave function amplitudes and distinguish different localization regimes. After describing an appropriate averaging procedure, we present our numerical results for the decay of the wave functions in Section V. We summarize our results in Section VI.

\section{THE ANDERSON MODEL}

We consider the Schrödinger equation in tight-binding approximation for the wave function of a quantum particle on regular lattices. The coefficients $\psi_{n}$ for each lattice site $n$ satisfy the tight-binding equation

$$
E \psi_{n}=\epsilon_{n} \psi_{n}+\sum_{\delta} V_{n, n+\delta} \psi_{n+\delta},
$$

where the sum runs over all nearest neighbor sites $n+\delta$ of site $n .\left|\psi_{n}\right|^{2}$ is the probability density on site $n$ for the wave function with energy $E$. The hopping terms $V_{n, n+\delta}$ are constant for nearest neighbor cluster sites and zero otherwise; for simplicity we take $V=1$ as energy unit. The values for the diagonal terms $\epsilon_{n}$ are randomly chosen from the interval $[-w / 2, w / 2]$, where $w$ is the disorder parameter (diagonal disorder).

Here, we consider the Anderson model with periodic as well as hard wall boundary conditions for a linear chain and on the square lattice In these cases the wave functions are known to be localized 3 , 17 even for small disorder strength $w$. For large distances $r$ from the localization center, the decay of the eigenfunction amplitudes is believed to be exponential. Thus, a localization length $\lambda$ and the Lyapunov exponent $\gamma \equiv \lambda^{-1}$ can be defined to describe the asymptotic decay of the mean logarithm of the amplitudes,

$$
\langle\ln |\psi(r) / \psi(0)|\rangle=-r / \lambda=-r \gamma
$$

for $r \gg \lambda$. In 1d the localization length in the band center $E \simeq 0$ can be calculated analytically from a recursion equation derived from Eq. (1) using Greens functions:18 $\lambda(w) \simeq$
$105.045 / w^{2}$. This result has also been verified numerically. 19 While the prefactor slightly depends on the energy $E$, the $1 / w^{2}$-dependence holds for all eigenstates except at the band edges, where a different analytical theory applies 20 In $2 d$ there is no analytical formula for the localization length $\lambda$. The numerical results published so far were obtained using finite size scaling combined with (a) transfer matrix calculations of the conductance 17 and (b) exact diagonalization and level statistics.21

We are interested in the distribution of the amplitudes $\left|\psi_{n}\right|$ for the eigenfunctions of Eq. (11). Because of the disorder, the eigenvalue equation has to be solved numerically. In 1d we use apiteration method,22 while we employ a Lanczos algorithm 23 in $2 \mathrm{~d}$.

\section{THE AMPLITUDE DISTRIBUTIONS}

\section{A. Definition}

In the original single-parameter scaling theoryd (SPST) the fluctuations of the wave functions had not been considered. An extension of the theory includes the scaling of the fluctuations. Still it has been assumed that there is only one relevant scaling parameter characterizing the localization behavior and the fluctuation behavior. This means that, e. g., the position of the center as well as the width of the distributions of conductances, Lyapunov exponents, or localization lengths can be characterized by only one scaling variable. Practically the distribution of the parameter

$$
\tilde{\gamma}(L)=\frac{1}{2 L} \ln (1+1 / g)
$$

with the conductance $g$ and the system size $L$ has been investigated theoreticaly 5 within the so-called random-phase hypothesis, see Ref. age $\langle\tilde{\gamma}(L)\rangle$ was shown 17 to approach the Lyapunov exponent $\gamma$

$$
\langle\tilde{\gamma}(L)\rangle=\gamma \equiv \lambda^{-1} \quad \text { for } \quad L \gg \gamma^{-1}
$$

its fluctuations were expected to become

$$
\left\langle\tilde{\gamma}^{2}(L)\right\rangle-\langle\tilde{\gamma}(L)\rangle^{2}=\gamma / L \quad \text { for } \quad L \gg \gamma^{-1}
$$

asymptotically. Thus, $\gamma$ fully characterizes the asymptotic scaling behavior of the average $\tilde{\gamma}(L)$ and its fluctuations. Although many numerical calculations of $\langle\tilde{\gamma}(L)\rangle$ [testing Eq. (†) ] have been reported, there is only little work devoted to the scaling behavior of the fluctuations and their relation to $\gamma$. Recently Deych et al 6 considered the Lloyd model (which is identical to the Anderson model with diagonal disorder except for a Cauchy distribution of the site energies) and found that states at the tail of the density of states violate the SPST and that there exists a new length scale that is responsible for this violation.

In this paper, we determine numerically the distribution of the logarithm of normalized amplitudes, $A(r) \equiv$ 
- $\ln |\psi(r) / \psi(0)|$, for large distances $r$ from the localization center (at $r=0$ ). The distance $r$ is directly related to the system size $L$ used in Eqs. (3)-(5), since the value and the fluctuations of the conductance $g$ for a system of size $L$ reflect the value and the fluctuations of its wave functions at the distance $r=L$ from the localization center. Following Eqs. (2) and (4), it is obvious that

$$
\langle A(r)\rangle \equiv\left\langle-\ln \left|\frac{\psi(r)}{\psi(0)}\right|\right\rangle=\frac{r}{\lambda}=r\langle\tilde{\gamma}(r)\rangle
$$

for $r \gg \lambda$. Hence, $A(r)$ corresponds to $r \tilde{\gamma}(r)$, and the fluctuations of $A(r)$ are expected to become

$$
\left\langle A^{2}(r)\right\rangle-\langle A(r)\rangle^{2}=\left(\left\langle\tilde{\gamma}^{2}\right\rangle-\langle\tilde{\gamma}\rangle^{2}\right) r^{2}=\gamma r=r / \lambda
$$

for large $r$ in the standard SPST with the random-phase hypothesis following Eq. (5). In order to estimate possible deviations from this standard single parameter scaling behavior, we will calculate the fluctuation parameter

$$
\sigma(r) \equiv 2\left[\left\langle A^{2}(r)\right\rangle-\langle A(r)\rangle^{2}\right] \lambda(r) / r
$$

which is expected to become $\sigma=2$ asymptotically in the standard SPST ${ }^{-}$according to Eq. (77) for the Anderson model and $\sigma=4$ for the Lloyd model. 8

In an alternative approach it has been shown some years ago, that the random matrix theory 24 can be applied to electropic eigenfunctions in disordered systems in $3 \mathrm{~d}^{25}$ and in $2 \mathrm{~d} .21$ In $2 \mathrm{~d}$, for small disorder strength $w$, the localization length becomes larger than the system size, $\lambda \gg L$, and the wave functions become quasi extended. In this regime, as well as in the metallic regime in $3 \mathrm{~d}$, the random matrix theory correctly describes the level spacing distribution as well as the distribution of the eigenfunction amplitudes $\left|\psi_{n}\right|$, which are usually assumed to be normalized according to $\sum_{n}\left|\psi_{n}\right|^{2}=1$ he intensity distribution (histogram) is usually defined as $11,12,14,15,26$

$$
f(t)=\left\langle\frac{1}{N} \sum_{n=1}^{N} \delta\left(t-\left|\psi_{n}^{\left(E^{\prime}\right)}\right|^{2} N\right)\right\rangle_{E},
$$

where the sum runs over all lattice sites, $N$ is the total number of lattice sites, and the average $\langle\cdots\rangle_{E}$ is taken over several eigenfunctions $\psi_{n}^{\left(E^{\prime}\right)}$ with energy $E^{\prime} \approx E$. In the limit of small disorder strength $f(t)$ is given by the universal Porter-Thomas distribution 27

$$
f_{\mathrm{PT}}(t)=\frac{1}{\sqrt{2 \pi t}} \exp (-t / 2)
$$

This result has been confirmed numerically recently for quasi extended states in the Anderson model in $2 \mathrm{~d}^{11}$ and for the metallic regime in $3 \mathrm{~d} .12 .15$

The intensity distribution $f(t)$, however, cannot appropriately describe the behavior of localized wave functions in the limit of strong localization $\lambda \ll L$. The analytical form of $f(t)$ obtained by inserting a simple exponential decay without any amplitude fluctuations into the definition (9) was shown to be consistent with the numerical results for real fluctuating Anderson wave functions.11 Thus, a simple exponential decay without any fluctuations cannot be distinguished from a real fluctuating wave function, if (9) is used to calculate the intensity statistics. The fluctuations simply do not affect $f(t)$. This indicates that the definition (9) is not appropriate to fully characterize localized eigenfunctions. Further, since no reference to the localization center is taken into account in (9), the calculation of the decay of mean wave functions is not possible from $f(t)$. Another problem is that $f(t)$ depends on the system size $N$ for localized states. So, we introduce a different definition for the amplitude distribution here, which avoids these problems.

Since we are interested in the decay of the localized wave functions, we investigate the distribution of the amplitudes $|\psi|$ at a certain distance $r$ from the localization center. By definition, the localization center for each eigenfunction $\left(\psi_{n}^{(E)}\right)$ is that site $n_{0}$, where the maximum of the amplitudes occurs, i. e. $\left|\psi_{n_{0}}^{(E)}\right|=\max _{n}\left|\psi_{n}^{(E)}\right|$. An alternative definition for the localization center would be to consider the lattice point most close to the "center of gravity" of the wave function as the center of localization. That is the site $n_{1}$ where $\sum_{n}\left[\left(x_{n}-x_{n_{1}}\right)^{2}+\left(y_{n}-y_{n_{1}}\right)^{2}\right]\left|\psi_{n}\right|^{2}$ reaches its minimum value ( $x_{n}$ and $y_{n}$ designate the coordinates of site $n$ here). Often, these two definitions of the localization center coincide. But even if they do not coincide, there is practically no difference in the results for the amplitude distributions, as we will show below. In order to achieve a system-size independent normalization of the amplitudes $|\psi|$, which allows us to compare numerical data for different system sizes and simplifies the analytical description significantly, we choose to set $\left|\psi_{n_{0}}\right|=1$. Note that this choice is different from the usual normalization $\sum_{n}\left|\psi_{n}\right|^{2}=1$ underlying the definition (9), since the sum is always larger than one for our normalization.

To describe the actual decay of the localized wave functions, we do not consider the distribution of the amplitudes for the whole eigenfunction [as in the definition (9)], but instead look at the distributions of the amplitudes at given distances $r$ from the localization center. Hence, we replace $f(t)$ by the histogram distribution function $H(A, r)$ defined by

$$
H(A, r)=\left\langle\frac{1}{N_{r}} \sum_{n=1}^{N} \delta\left(r-r_{n}\right) \delta\left(A+\ln \left|\psi_{n}^{\left(E^{\prime}\right)}\right|\right)\right\rangle_{E}
$$

where $r_{n}$ is the distance of the site $n$ from the localization center, and $N_{r}$ is the number of sites with $r_{n}=r$, i. e. $N_{r}=\sum_{n=1}^{N} \delta\left(r-r_{n}\right)$. In the definition (11), we use the variable $A \equiv-\ln \left|\psi_{n}^{\left(E^{\prime}\right)}\right|$ instead of $t \equiv\left|\psi_{n}^{\left(E^{\prime}\right)}\right|^{2} N$ from 9 for two reasons: (i) $t$ is system-size dependent for localized states, and (ii) $A$ can be related much more easily to the predictions of the standard SPST, that we want to test. In fact, we have already derived the relation in Eqs. (6) and (7). Note that $H(-\ln |\psi|, r)$ is the probability density of finding the amplitude $|\psi|$ at distance $r$ from the localization center. In the following, we will use this definition of the eigenfunction amplitude distributions to present our numerical results and discuss the localization behavior. 


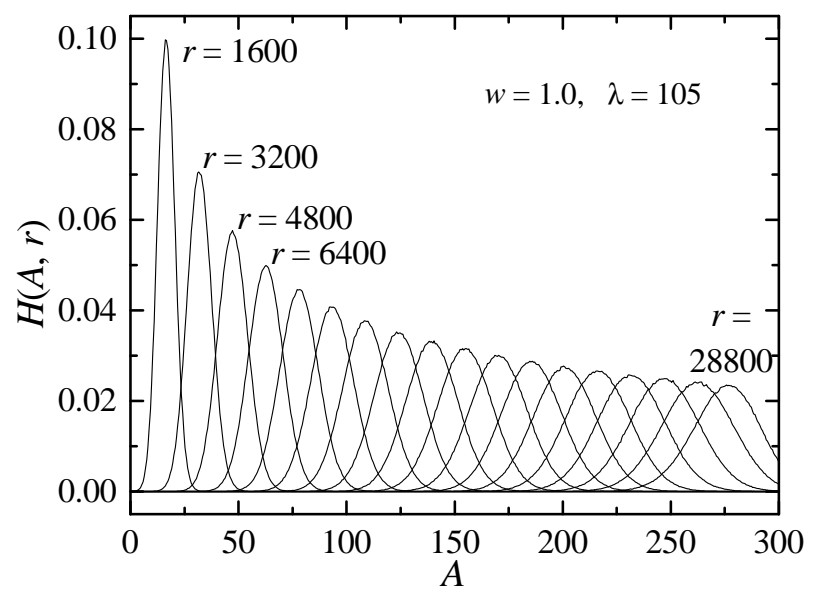

FIG. 1.: Histogram distributions $H(A, r)$ of the absolute amplitude values $|\psi|$ at distance $r$ from the localization center versus $A \equiv-\ln |\psi|$ for electronic wave functions in the Anderson model in 1d with disorder strength $w=1.0$ at several fixed distances $r$ from the center of localization ranging from $r=1600$ (leftmost curve) with increment $\Delta r=1600$ to $r=28800$ (rightmost curve). To determine the distributions, more than $10^{6}$ eigenfunctions have been calculated on chains of lengths $L=30000$.

\section{B. Results in 1d}

Figures 11 and 2 show our numerical results of the amplitude distribution functions $H(-\ln |\psi|, r)$ for the Anderson model in $1 \mathrm{~d}$ with periodic boundary conditions. Using an iteration method22 we have calculated eigenfunctions with energy $E \approx 0$ (corresponding to the band center) for linear chains with five different disorder strengths $w$ and determined the distribution functions $H(A, r)$ for several values of $r$. Figure 11 shows 18 of these histogram distributions calculated for more than $10^{6}$ chains of length $L=30000$ with $w=1.0$.

We find that the histograms $H(-\ln |\psi|, r)$ of the amplitudes $|\psi|$ at fixed distances $r$ from the localization center surprisingly well follow a log-normal ansatz,

$$
H_{\ln }(-\ln |\psi|, r) \equiv \sqrt{\frac{\lambda}{\pi \sigma r}} \exp \left[-\frac{(\ln |\psi|+r / \lambda)^{2}}{\sigma r / \lambda}\right] .
$$

In general, both parameters, $\lambda$ and $\sigma$, might depend on $r$. Since $H_{\ln }(A, r)$ is a simple Gaussian distribution with average value $\langle A(r)\rangle=\int A H_{\ln }(A, r) d A=r / \lambda$ and variance $\left\langle A^{2}(r)\right\rangle-\langle A(r)\rangle^{2}=\sigma r / 2 \lambda$, the two parameters $\lambda$ and $\sigma$ correspond to the localization length and the fluctuation parameter, respectively, according to Eqs. (6) and (8). Note that the amplitude $|\psi|=\exp (-r / \lambda)$ occurs with the largest probability at distance $r$ from the center of localization, which further prompts us to identify the parameter $\lambda$ with the localization length. In the following, $\lambda(r)$ always refers to the parameter determined from the distribution $H(A, r)$ for a given $r$, while $\lambda$ represents the asymptotic value of $\lambda(r)$.

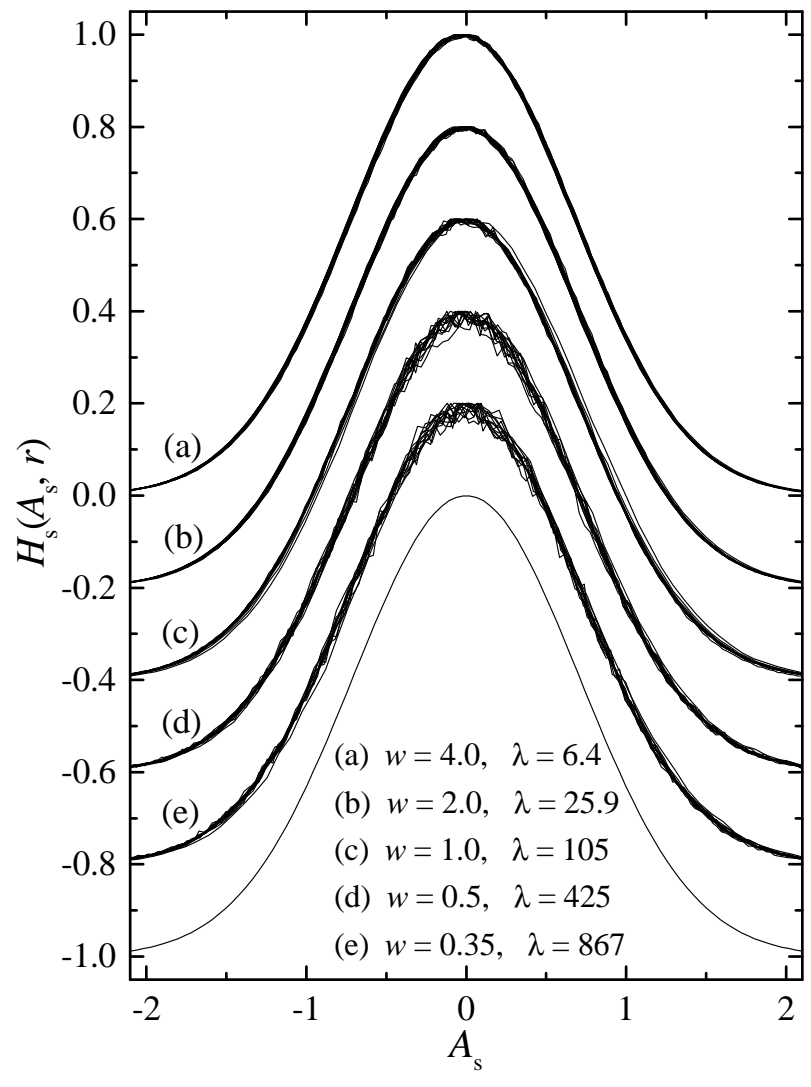

FIG. 2.: Scaled distributions $H_{\mathrm{s}}\left(A_{\mathrm{s}}, r\right) \equiv H(A, r) \sqrt{\pi \sigma r / \lambda}$ of the amplitude values $|\psi|$ at distance $r$ from the localization center versus $A_{\mathrm{s}} \equiv(A-r / \lambda) / \sqrt{\sigma r / \lambda}$ for electronic wave functions in the Anderson model in $1 \mathrm{~d}$ with disorder strength $w=4.0,2.0,1.0$, 0.5 , and 0.35 at several distances $r$ from the center of localization ranging from $r \approx 20 \lambda$ to $250 \lambda$. Due to the scaling the data collapse onto single curves. The curves for different $w$ have been shifted by multiples of 0.2. The continuous line below the data corresponds to the ansatz Eq. (12). To determine the distributions, (a) 4,200,000, (b) $3,400,000$, (c) $1,100,000$, (d) 200,000 and (e) 170,000 eigenfunctions have been calculated on chains of lengths $L>250 \lambda(w)$.

The log-normal distribution of the amplitudes can be motivated by the following argument. If $1 \mathrm{~d}$ wave functions are calculated using the transfer matrix method, the value of $\psi_{n}$ for large $n$ is related to the values of $\psi_{n}$ for the two initial sites, $n=0$ and $n=1$, by the product of $n$ transfer matrixes $M_{m}$,

$$
\left[\prod_{m=n}^{1} M_{m}\right]\left(\begin{array}{l}
\psi_{1} \\
\psi_{0}
\end{array}\right)=\left(\begin{array}{c}
\psi_{n+1} \\
\psi_{n}
\end{array}\right), \quad M_{m}=\left(\begin{array}{cc}
E-\epsilon_{m} & -1 \\
1 & 0
\end{array}\right) .
$$

This product of matrices will involve many products of the random site energies $\epsilon_{n}$. Products of many independently distributed random numbers asymptotically follow a log-normal distribution according to the central limit theorem (under some conditions, which we do not want to discuss in detail here). Thus, it is reasonable that $\psi_{n}$ will also follow a lognormal distribution asymptotically for large $n$. 
The agreement between the numerical distributions $H(A, r)$ (shown in Fig. 1) and the log-normal distribution $H_{\mathrm{ln}}$ [Eq. (12)] becomes perfect for large $r$ values. This indicates that the distribution $H(A, r)$ follows Eq. (12) asymptotically. In addition, we find that both parameters, $\lambda$ and $\sigma$, are independent of $r$. Fits of Eq. (12) to the numerical distributions $H(A, r)$ for $w=1.0$ shown in Fig. 1 give $\lambda=105 \pm 1$ and $\sigma=2.1 \pm 0.15$. The value of $\lambda$ is in perfect agreement with the analytical result $18 \lambda(w) \simeq 105.045 / w^{2}$. The value of $\sigma$ is consistent with $\sigma=2$ expected in the standard SPST.

Since both parameters do not depend on $r$, the distributions shown in Fig. 1 can be rescaled in order to obtain a data collapse for the different distances $r$ from the localization center. If we multiply $H(A, r)$ by $\sqrt{\pi \sigma r / \lambda}$ and replace $A$ by $A_{\mathrm{s}} \equiv(A-r / \lambda) / \sqrt{\sigma r / \lambda}$, all distribution will collapse upon each other. This is shown in Fig. 2, where data for the other disorder strengths $w$ we considered are also included. Since the dependence of the localization length $\lambda$ on $w$ is known, and the fluctuation parameter $\sigma$ turns out to be independent of $w$, we are able to rescale the distributions for all $r$ and $w$ in order to obtain a full data collapse also for the other disorder strengths. The numerical result $\sigma=2.1 \pm 0.15$, which is consistent with $\sigma=2$, fully confirms the standard SPST for the Anderson model in $1 \mathrm{~d}$.

\section{Results in 2d}

For the Anderson model in $2 \mathrm{~d}$ the scaling behavior of the histograms of amplitudes $|\psi|$ at fixed (Euclidian) distance $r$ from the localization center turns out to be not as simple as in 1d. Using the Lanczos algorithm 23 we have calculated eigenfunctions of Eq. (11) for energies $E \approx 0$ and two disorder strengths $w=8.5$ and $w=10.0$. For these values of $w$ the amplitudes of the localized states decay roughly to $10^{-13}$ and $10^{-20}$, respectively, on our $300 \times 300$ square lattice. This allows to observe the localization effects over several orders of magnitude. Quadruplicate precision is used in all calculations in order to get reliable values also for small amplitudes $|\psi| \approx 10^{-20}$. We compare the results for periodic boundary conditions (PBC) and hard wall boundary conditions (HWBC).

We find that the histogram distributions $H(A, r)$ can still be well approximated by the log-normal distribution $H_{\ln }(A, r)$ [Eq. (12)]. But now the parameter $\lambda(r)$ that corresponds to the localization length clearly depends on $r$. This dependence is shown in the main part of Fig. 3 , where $\lambda$ is plotted versus $r$ on a log-linear scale for systems with the disorder strength $w=10.0$ and PBC as well as for $w=8.5$ and HWBC. We also checked $w=8.5$ with $\mathrm{PBC}$ and $w=10.0$ with HWBC and found no significant alterations in the behavior. Further, the results do not change if we disregard all those eigenfunctions that did not reach a preset accuracy limit in the Lanczos calculations or select only eigenfunctions within a reduced energy interval. The results also do not depend on the way the center of localization is defined (as maximum or "center of gravity", see Section III.A).

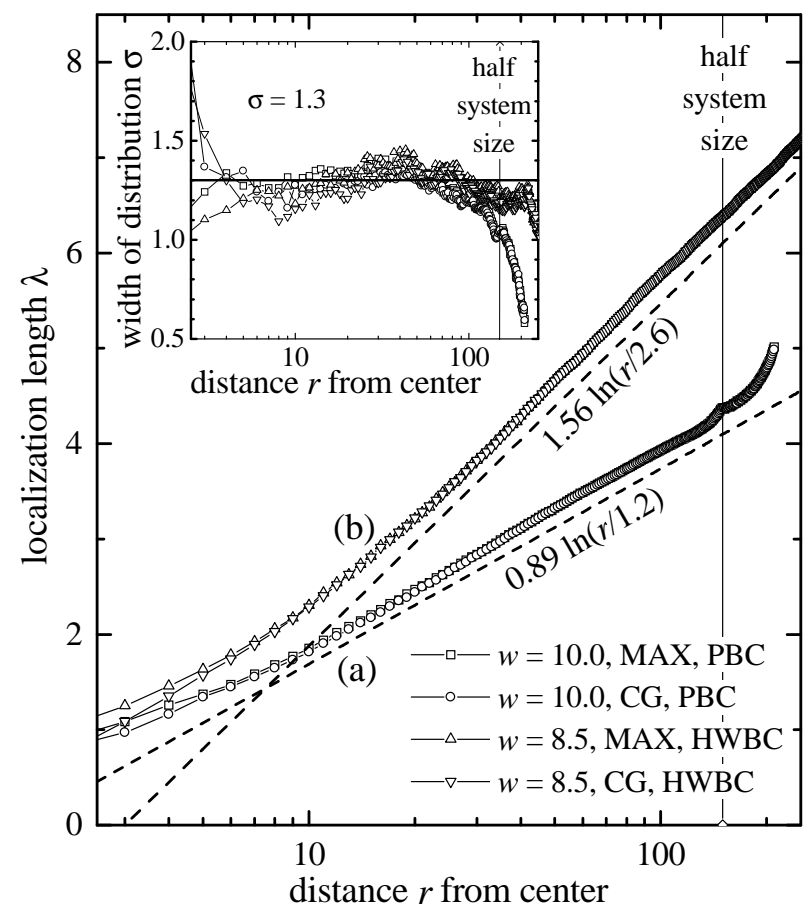

FIG. 3.: Localization length $\lambda(r)$ of Anderson wave functions in $2 \mathrm{~d}$ in the localized regime for (a) $w=10.0$ and (b) $w=8.5$. The values of $\lambda(r)$ have been determined by fits of Eq. (12) to the amplitude distributions $H(A, r)$ for each distance $r$ from the localization center. Data for periodic boundary conditions (PBC, a) as well as hard-wall boundary conditions (HWBC, b) have been analyzed. The results for both definitions of the localization center [as the maximum (MAX) and as the "center of gravity" (CG)] are practically identical (except for $r<10$ ) and well described by a logarithmic dependence of $\lambda$ on $r$ : (a) $\lambda(r) / 0.89=\ln (r / 1.2)$ and (b) $\lambda(r) / 1.56=\ln (r / 2.6)$. The straight lines correspond to these fits, but they are shifted by a constant offset for visibility. In the inset, the estimated fluctuation parameters $\sigma(r)$ of the distributions are shown. They are approximately independent of $r$ and $w$. We find $\sigma=1.3 \pm 0.2$. For both disorder strengths, about 1000 eigenfunctions were calculated with the Lanczos algorithm with quadruplicate precision on a $300 \times 300$ lattice.

The values of $\lambda(r)$ and $\sigma(r)$ shown in Fig. B have been obtained by fitting Eq. $(12)$ to $H(A, r)$ for each $r$. Finite-size effects are clearly observable for $\mathrm{PBC}$, while they seem to be rather negligible for $\mathrm{HWBC}$. The rather strong finite-size effects in the case of PBC are probably due to the many additional paths leading from the localization center to a remote site via the edges of the lattice. In all cases the $\lambda(r)$ dependence is clearly a logarithmic one for $r>10$ :

$$
\lambda(r) / c_{\lambda}=\ln \left(r / c_{r}\right)
$$

where the two parameters $c_{\lambda}$ and $c_{r}$ describe the scales of the localization length $\lambda$ and the distance $r$, respectively. Only for small $r$ values (i. e. $r \ll 20$ ), a power-law fit is also possible. The logarithmic dependence is observed over more than one order of magnitude in $r$ in all our numerical simulations. 


\begin{tabular}{c|c|c|c|c|c|c}
$w$ & BC & $E_{\max }$ & number & $c_{\lambda}$ & $c_{r}$ & $\sigma$ \\
\hline 8.5 & HWBC & 0.05 & 994 & 1.56 & 2.6 & 1.35 \\
8.5 & HWBC & 0.005 & 359 & 1.54 & 2.5 & 1.34 \\
8.5 & HWBC & 0.001 & 76 & 1.53 & 2.4 & 1.28 \\
\hline 8.5 & PBC & 0.05 & 227 & 1.64 & 3.1 & 1.27 \\
8.5 & PBC & 0.005 & 73 & 1.68 & 3.2 & 1.37 \\
\hline 10.0 & HWBC & 0.05 & 288 & 0.91 & 1.3 & 1.32 \\
10.0 & HWBC & 0.005 & 97 & 0.92 & 1.4 & 1.34 \\
\hline 10.0 & PBC & 0.05 & 1029 & 0.89 & 1.2 & 1.25 \\
10.0 & PBC & 0.005 & 309 & 0.90 & 1.2 & 1.24 \\
10.0 & PBC & 0.001 & 68 & 0.92 & 1.4 & 1.20
\end{tabular}

TABLE 1: The values of $c_{\lambda}$ and $c_{r}$ from fits of Eq. 14) to the localization length $\lambda(r)$ as well as the distribution width $\sigma$ for the Anderson model in $2 \mathrm{~d}$. The results for two disorder strengths $w=8.5$ and $w=10.0$, for both types of boundary conditions (BC, HWBC $=$ hard-wall, $\mathrm{PBC}=$ periodic boundary conditions), and for different subsets of the eigenfunctions are reported. The value of $E_{\max }$ determines the width of the energy interval $\left[0, E_{\max }\right]$ for the selected eigenfunctions. The number of eigenfunctions involved in each fit is also reported. The fitting range is $30>r>120$ in all cases, and the maximum has been used to define the localization center.

Table 1 reports the fitted values of $c_{\lambda}$ and $c_{r}$ for both disorder strengths, $w=8.5$ and $w=10.0$, for both types of boundary conditions, and for different subsets of the eigenfunctions. The value of $E_{\max }$ determines the width of the energy interval $\left[0, E_{\max }\right]$ for the selected eigenfunctions. The table shows that $c_{\lambda}$ and $c_{r}$ clearly depend on $w$, but not on the width of the energy interval. The values for different boundary conditions are also very close to each other, and we cannot find any systematic dependence upon the boundary conditions. Both, $c_{\lambda}$ and $c_{r}$, are rather close to the microscopic distance between the sites (set to one here), but they are definitely not simply identical to the microscopic distance, since both depend strongly on the disorder parameter $w$. The significant logarithmic dependence of the localization length $\lambda(r)$ on the distance $r$ from the localization center may explain the somewhat different numerical resulsfor the localization length that have been obtained before.1.21 When comparing with those results one has to keep in mind, though, that they were determined using finite size scaling of the conductance and level statistics, respectively, while our determination does not involve finite size scaling. In the finite size scaling techniques, one value of the localization length $\lambda_{L}$ or a similar level statistics parameter is calculated for each configuration. Then the average finite size parameters are extrapolated to infinite system size by rescaling the results for different disorder $w$ in a scaling plot, assuming the existence and scaling behavior of $\lambda_{\infty}$. Here, in contrast, we do not assume any scaling properties of the localization length and determine the dependence of $\lambda(r)$ upon the distance $r$ from the origin for each system.

In contrast to $\lambda(r)$, the parameter $\sigma$ characterizing the width of the amplitude distributions and shown in the inset of Fig. 3 is still independent of $r$, except for the pronounced finite-size effects in the case of PBC. We find $\sigma=1.3 \pm 0.2$ for both disorder strengths $w$, both types of boundary conditions, and all selections of eigenfunctions as reported in Table 1. This value clearly deviates from $\sigma=2$ expected for the standard SPST.

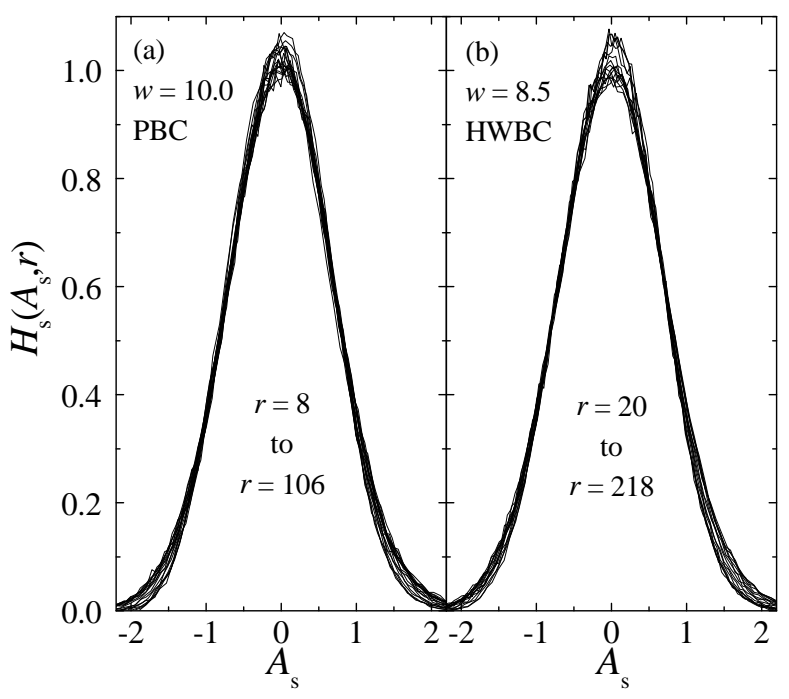

FIG. 4.: Scaled distributions $H_{\mathrm{s}}\left(A_{\mathrm{s}}, r\right)=H(A, r) \sqrt{\pi \sigma r / \lambda(r)}$ of the amplitude values $|\psi|$ at (Euclidian) distance $r$ from the localization center versus $A_{\mathrm{s}}=[A-r / \lambda(r)] / \sqrt{\sigma r / \lambda(r)}$ for electronic wave functions in the Anderson model in $2 \mathrm{~d}$ with disorder strength (a) $w=10.0$ (PBC) and (b) $w=8.5$ (HWBC) at several fixed distances $r$ from the center of localization, (a) $r=8, \ldots, 106$, and (b) $r=20, \ldots, 182$. The $r$-dependent localization lengths $\lambda(r)$ for the rescaling procedure have been taken from logarithmic fits in Fig. 3, and the width parameter $\sigma$ is fixed to 1.35 . For each part of the figure, $\approx 1000$ eigenfunctions were calculated using the Lanczos algorithm with quadruplicate precision on a $300 \times 300$ lattice.

Using the logarithmic $\lambda(r)$ dependence, Eq. 14), with the parameters from Table 1 the amplitude distribution functions $H(A, r)$ for the Anderson model in $2 \mathrm{~d}$ can be rescaled to obtain a data collapse similar to Fig. 2. The result is shown in Fig. 4, again for $w=10.0$ with $\mathrm{PBC}$ and $w=8.5$ with HWBC. The numerical results confirm the log-normal shape of the amplitude distribution $H(-\ln |\psi|, r)$.

In summary, we find two major deviations from the standard SPST for the Anderson model in 2d: (i) The logarithmic $\lambda(r)$ dependence indicates that an asymptotic value $\lambda \equiv \gamma^{-1}$ (assumed in the SPST) might be ill defined, since the logarithmic function does not converge. (ii) The fluctuations still follow the SPST hypothesis Eq. (7), if $\lambda$ is replaced by the $r$-dependent localization length $\lambda(r)$ in Eq. (14), and an additional prefactor $\sigma / 2$ is inserted. But since this prefactor is smaller than one, the fluctuations in the Anderson model in $2 \mathrm{~d}$ are smaller than those predicted by the standard SPST hypothesis.

In order to find out, if these two types of deviations from the standard SPST are a generic feature of any system in $2 d$, we have determined the amplitude distributions and the values of $\lambda$ and $\sigma$ for electronic eigenfunctions on percolation clusters at the critical concentration, which we had studied in a previous work 28 Percolation 9 is a standard model for structurally disordered solids. For site percolation, the Schrödinger equa- 
tion in tight-binding approximation is identical to Eq. (11) with $\epsilon_{n}=0$ for occupied sites (concentratipn $p$ ), and $\epsilon_{n}=\infty$ for unoccupied sites (concentration $1-p$ ) 29 The electronic wave functions are known to be localized for percolation clusters at the critical concentration $p=p_{\mathrm{c}}$ for any embedding dimension. We found that the amplitude distributions at given topological distances from the localization center again obey the log-normal distribution (12) for clusters in $2 \mathrm{~d}$ as well as in $3 \mathrm{~d}$. A data collapse is obtained with constant parameter $\lambda$, and no logarithmic dependence of $\lambda$ on the distance is observed. Thus, the first deviation from the standard SPST that we observed in the $2 \mathrm{~d}$ Anderson model does not show up for the percolation model. Nevertheless, the second deviation, the modified value of the fluctuation parameter $\sigma$, also occurs in percolation. But in contrast to the reduced value $\sigma=1.3$ for the Anderson model in $2 \mathrm{~d}$ (compared with the standard SPST value $\sigma=2$ ), we find increased values $\sigma \approx 2.6$ and 3.0 for percolation in $2 \mathrm{~d}$ and $3 \mathrm{~d}$, respectively, which are still smaller than $\sigma=4$ for the Lloyd model. We conclude that a model dependent second parameter $\sigma$ is required to describe the scaling behavior of the amplitude fluctuations.

\section{ANALYTICAL RESULTS FOR THE MEAN WAVE FUNCTION}

Since we can very well approximate the distribution of eigenfunction amplitudes $|\psi|$ at large distances $r \gg \lambda$ by the log-normal distribution (12), we can directly calculate the decay of the average wave function $\langle|\psi(r)|\rangle$ for large $r$ by simple integration. We can also estimate the way this average, which we denote by $\Psi_{N}(r)$, depends on the number of averaged configurations $N$. In particular, we can obtain $\Psi_{N}(r)$ in the asymptotic regime which is not accessible numerically. The calculation is valid for Anderson wave functions in $1 \mathrm{~d}$ and $2 \mathrm{~d}$ (as well as for the percolation model), since the same lognormal distribution $H_{\ln }(A, r)$, applies to all cases and only the two parameters $\lambda$ and $\sigma$ have to be adapted. Note, that the $r$ dependent localization length $\lambda(r)$ from Fig. 2 has to be used for the Anderson model in $2 \mathrm{~d}$, while $\lambda$ is constant otherwise.

In order to describe the localization behavior properly, we are led to the ansatz

$$
\ln \Psi_{N}(r) \sim-r^{d \Psi}
$$

where $d_{\Psi}$ is an effective localization exponent. If we average over all configurations, the resulting quantity $\Psi_{\infty}(r)$ is related to the distribution $H(A, r)$ by

$$
\Psi_{\infty}(r)=\langle|\psi(r)|\rangle=\int_{0}^{\infty} \exp (-A) H(A, r) d A
$$

with $A \equiv-\ln |\psi|$. Note that only positive values of $A$ are possible because of our normalization $\psi_{n_{0}}=1$ for the localization center (maximum) at site $n_{0}$. In the preceeding Section we have numerically shown that $H(A, r)$ can be well approximated by the log-normal distribution $H_{\ln }(A, r)$ for large distances $r$ from the localization center, where the amplitudes $|\psi|$ are small and $A \equiv-\ln |\psi|$ large. Thus, we insert $H_{\ln }(A, r)$ for $H(A, r)$ in Eq. (16). However, the exact shape of $H(A, r)$ for small distances, corresponding to large amplitudes, is not known exactly. Hence, our result will be exact only for large values of $r$. We further have to assume that the number of configurations $N$ is finite, so that the extreme tails of the distribution $H(A, r)$, i. e. the very rare events possibly involving small $A$, are not important. Our treatment includes a description of the typical average over one configuration (one eigenfunction), which corresponds to $N=1$.

For a finite number $N$ of configurations, the total number of sites at distance $r$ from the localization center is identical to $N N_{r}$ with $N_{r}=a r^{d-1}$ and $a=2(2 \pi)$ for the Anderson model in $1 \mathrm{~d}(2 \mathrm{~d})$. Clearly, those values of $|\psi|$ with a too small probability, i. e. probability smaller than $1 /\left(N N_{r}\right)$, are unlikely to occur in $N$ typical configurations. Thus, we must limit the distribution $H_{\ln }(A, r)$ by cutting off the area $1 /\left(2 N N_{r}\right)$ at each of the two tails. According to Eq. (12) the area below a lower cutoff $A_{\min }$ is given by

$$
\int_{-\infty}^{A_{\min }} H_{\ln }(A, r) d A=\frac{1}{2}\left\{1+\operatorname{erf}\left[\left(A_{\min }-\frac{r}{\lambda}\right) \sqrt{\frac{\lambda}{\sigma r}}\right]\right\}
$$

where $\operatorname{erf}(x) \equiv 2 \pi^{-1 / 2} \int_{0}^{x} \exp \left(-t^{2}\right) d t$ is the error function. Equating this area to $1 /\left(2 N N_{r}\right)=\left(2 N a r^{d-1}\right)^{-1}$ and using the inverse error function $\operatorname{erfinv}(x)$ we obtain the lower cutoff value

$$
A_{\min }=\max \left\{0, \frac{r}{\lambda}-\sqrt{\frac{\sigma r}{\lambda}} \operatorname{erfinv}\left(1-\frac{1}{N a r^{d-1}}\right)\right\}
$$

which replaces the lower integration bound in Eq. (16). Hence, for finite $N$, Eq. (16) becomes

$$
\Psi_{N}(r)=\int_{A_{\min }(r, N)}^{\infty} \exp (-A) H_{\ln }(A, r) d A
$$

The integration can be performed straightforwardly and gives

$$
\begin{array}{r}
\Psi_{N}(r)=\frac{1}{2} \exp \left[-\left(1-\frac{\sigma}{4}\right) \frac{r}{\lambda}\right] \times \\
\left\{1-\operatorname{erf}\left[\sqrt{\frac{\sigma r}{4 \lambda}}-\operatorname{erfinv}\left(1-\frac{1}{N a r^{d-1}}\right)\right]\right\} .
\end{array}
$$

The first term of this equation seems to indicate a regular exponential decay, if the second term could be disregarded and $\lambda$ was constant. But, indeed, the second term, involving the error function as well as a complicated dependence on the number of configurations $N$, cannot be disregarded. Thus, the average wave function $\Psi_{N}(r)$ depends explicitly on $N$ and exhibits significant deviations from the exponential from. 


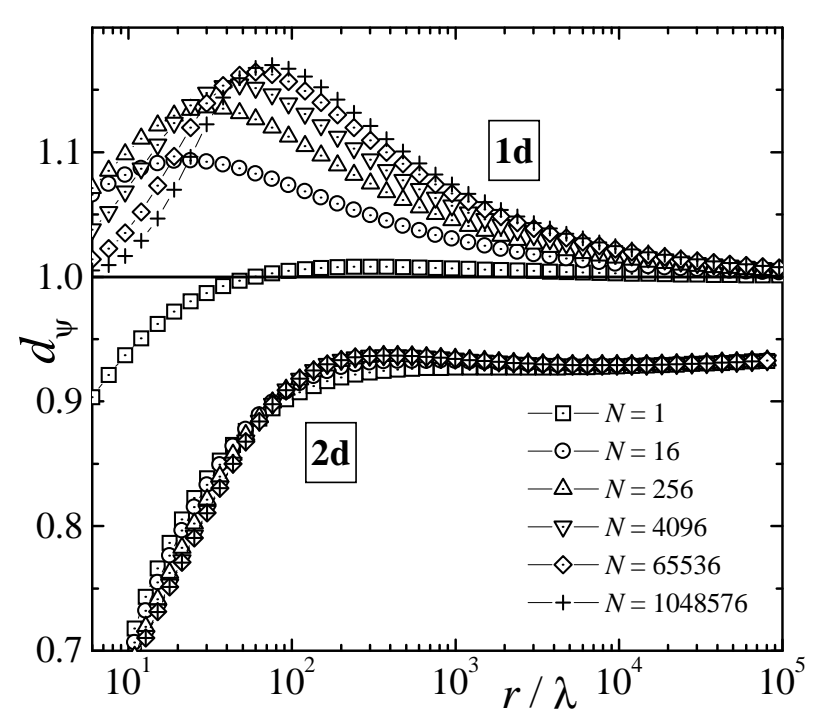

FIG. 5.: The theoretical effective localization exponents $d_{\Psi}$ determined by fits of the ansatz (15) to the analytical result (20) are shown versus $r / \lambda$ for several numbers of averaged configurations $N$ (see legend). For the $1 \mathrm{~d}$ results (upper curves) the parameters $\lambda=105$ and $\sigma=2$ have been used, while $\lambda(r) / 0.89=\ln (r / 1.2)$ (from Fig. 3) and $\sigma=1.3$ for the $2 \mathrm{~d}$ case. In the $1 \mathrm{~d}$ case we find strong dependence on $N$ and $d_{\Psi}>1$, indicating superlocalization, while $d_{\Psi}<1$ indicating sublocalization is observed in the $2 \mathrm{~d}$ case.

Equation (20) is supposed to be rigorous for sufficiently large $r$ values, where the distribution of $|\psi|$ is exactly described by the log-normal distribution $H_{\ln }(A, r)$. The decay of $\Psi_{N}(r)$ can be approximated by Eq. (15) with an effective localization exponent $d_{\Psi}$, which depends on $r$ and $N$. In Fig. 5, we trace $d_{\Psi}$ as a function of $r / \lambda$ for several values of $N$ using the parameters $\lambda(r)$ and $\sigma$ corresponding to the Anderson model in $1 \mathrm{~d}$ and $2 \mathrm{~d}$. We determined the values of $d_{\Psi}$ by local fits of Eq. (15) to Eq. (20). Surprisingly, the behavior is very different in $1 \mathrm{~d}$ and $2 \mathrm{~d}$.

In $1 \mathrm{~d}$, we find that the effective exponent $d_{\Psi}$ in the exponential ansatz (15) is larger than one except for the typical average $(N=1)$ which we will discuss later. The localization is stronger than the usual exponential localization. Thus, this regime can be described by the term "superlocalization". Furthermore, the values of $d_{\Psi}$ strongly depend on the number of configurations $N$. We observe a local maximum in the dependence of $d_{\Psi}$ on $r$ for $r \approx 10 \lambda$ to $100 \lambda$. While the height of the maximum increases with increasing $N$, indicating stronger superlocalization for an increasing number of configurations, the position of the maximum is shifted towards larger $r$ values. Only in the asymptotic limit for extremely large $r$ values, $r \approx 10^{5} \lambda$, the effective localization exponent $d_{\Psi}$ converges to one, yielding the theoretically predicted simple exponential behavior.

The typical average $\Psi_{1}(r)$, describing the behavior of one typical eigenfunction, can be deduced by setting $N=1$. As can be seen in Fig. 5, the exponent $d_{\Psi}$ is smaller than one for intermediate distances $r$ from the localization center (for $r<60 \lambda$ ). For larger $r$ it is very close to, but slightly above one. Practically, this behavior can no longer be distinguished from simple exponential localization for $r>50 \lambda$.

In $2 \mathrm{~d}$, however, the localization behavior appears to be remarkably different. As can be seen in Fig. 5, we observe hardly any $N$-dependence of the effective localization exponent $d_{\Psi}$, although the absolute value of the average wave function does slightly depend on $N$. The local maximum of $d_{\Psi}$ is so weak, that it will be practically invisible. The most important difference to the localization behavior in 1d, though, is the small value of $d_{\Psi}$. The effective localization exponent is smaller than one not only for intermediate $r$ values, but even remains below one asymptotically up to at least $r=10^{5} \lambda$. Thus simple exponential behavior of the average wave function can apparently not be reached for any practical system size. This is mainly due to the logarithmic dependence of the localization length $\lambda$ on $r$, that we have reported in Fig. 3 and inserted into Eq. (20) to derive $d_{\Psi}$. Consequently, this regime is not characterized by superlocalization as in $1 \mathrm{~d}$. We rather find a stretched exponential decay, so that the term "sublocalization" seems to be appropriate, because the localization behavior is weaker than simple exponential.

Equation (20) is not valid for small $r$ values where the real distribution $H(A, r)$ deviates from the log-normal form $H_{\ln }(A, r)$. Thus, deviations occur for $r$ approximately smaller than $10 \lambda$. Further deviations occur for intermediate $r$ and large $N$ values when the tails of the distribution become more important. Then the lower integration limit $A_{\min }(r, N)$ in Eq. (19) tends to zero and the distribution of rare events of wave function amplitudes $|\psi|$ close to the maximum (corresponding to $A \equiv-\ln |\psi|$ close to zero) becomes important.

Despite of this, the log-normal approximation $H_{\ln }(A, r)$ can be used for obtaining a qualitative picture of $\Psi_{N}(r)$ also at small $r$ values, where $A_{\min }(r, N)=0$ in Eq. (18). We introduce the cutoff at $A=0$, since no amplitude $\psi_{n}$ at distance $r>0$ from the localization center $n_{0}$ can be larger than $\left|\psi_{n_{0}}\right|=1$, and thus $A \equiv-\ln \left|\psi_{n}\right|>0$. It can be shown numerically, that the integration of Eq. (16) yields an effective localization exponent much smaller than one, $d_{\Psi} \approx 0.5$, for small $r$ in the ansatz Eq. (15). Thus, the decay is weaker than exponential in the small- $r$ regime, and we expect a regime of sublocalization. With increasing $N, A_{\min }(r, N)$ in Eq. (19) decreases reaching zero also for larger $r$ values. Thus, the small- $r$ regime with $A_{\min }(r, N)=0$ becomes dominant for averages over a large number of configurations. Since the lower integration limit becomes zero in Eq. (19) for the small$r$ regime, it is evident that $\Psi_{N}(r)$ does not depend on $N$.

\section{NUMERICAL RESULTS FOR THE MEAN WAVE FUNCTION}

\section{A. Averaging procedure}

Next we determine numerically the way the average amplitude values $|\psi(r)|$ decay with increasing distance $r$ from the localization center The mecessary averaging procedure consists of three steps.28 31, 22 


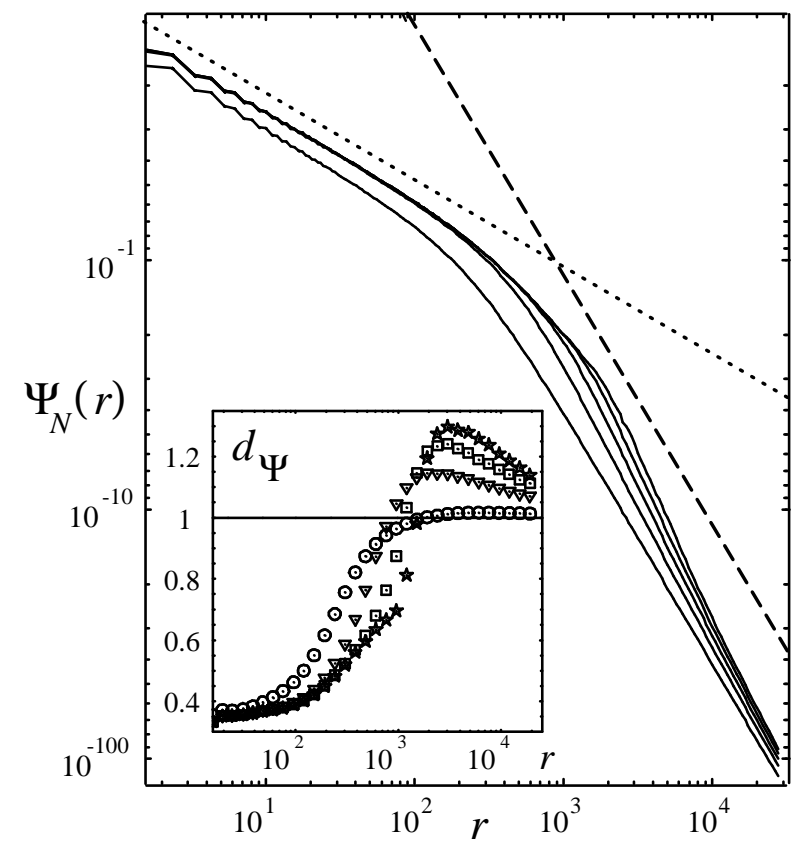

FIG. 6.: The decrease of the typical average amplitudes for $N$ configurations, $\Psi_{N}(r)$, versus $r$ for electronic wave functions in the Anderson model in $1 \mathrm{~d}$ for $w=1.0$ and $N=1,16,256,4096$, and 65536 (from the bottom to the top). For the plot, more than $10^{6}$ eigenfunctions have been calculated on chains of lengths $L=$ 30000. The straight lines with the slopes $d_{\Psi}=1$ (dashed) and $d_{\Psi}=0.4$ (dotted) are shown for comparison. In the inset, the effective local exponents $d_{\Psi}$ determined numerically from the slopes of the curves are shown versus $r$. The symbols correspond to the effective numbers of configurations, $N=1$ (circles), 16 (triangles), 256 (squares), and 4096 (stars).

1. In the first step we average, for each eigenfunction $\psi_{n}^{(\nu)}$ separately, the values of $\left|\psi_{n}^{(\nu)}\right|$ for all sites $n$ at given distances $r$ from the localization center. The resulting function $\psi^{(\nu)}(r)$ characterizes the spatial decrease of the $\nu$ th eigenfunction.

2 . In the second step, for obtaining the mean spatial decrease of $N$ eigenfunctions, we average $\psi^{(\nu)}(r)$ over $N$ configurations (eigenfunctions),

$$
\langle\psi(r)\rangle_{N} \equiv \frac{1}{N} \sum_{\nu=1}^{N} \psi^{(\nu)}(r) .
$$

If the system is not selfaveraging (which we find is the case here), the resulting values for $\langle\psi(r)\rangle_{N}$ will depend on the special set of $N$ configurations considered and will fluctuate from set to set.

3. To obtain the typical value of $\langle\psi(r)\rangle_{N}$, we are led, in the third step, to the logarithmic average over many sets of $N$ configurations, i. e wererage $\ln \langle\psi(r)\rangle_{N}$ over many sets of $N$ eigenfunctions, 26, , 1,32

$$
\Psi_{N}(r) \equiv \exp \left\langle\ln \langle\psi(r)\rangle_{N}\right\rangle .
$$

In the following, we shall discuss exclusively this "typical average over $N$ configurations" defined by Eq. (22).

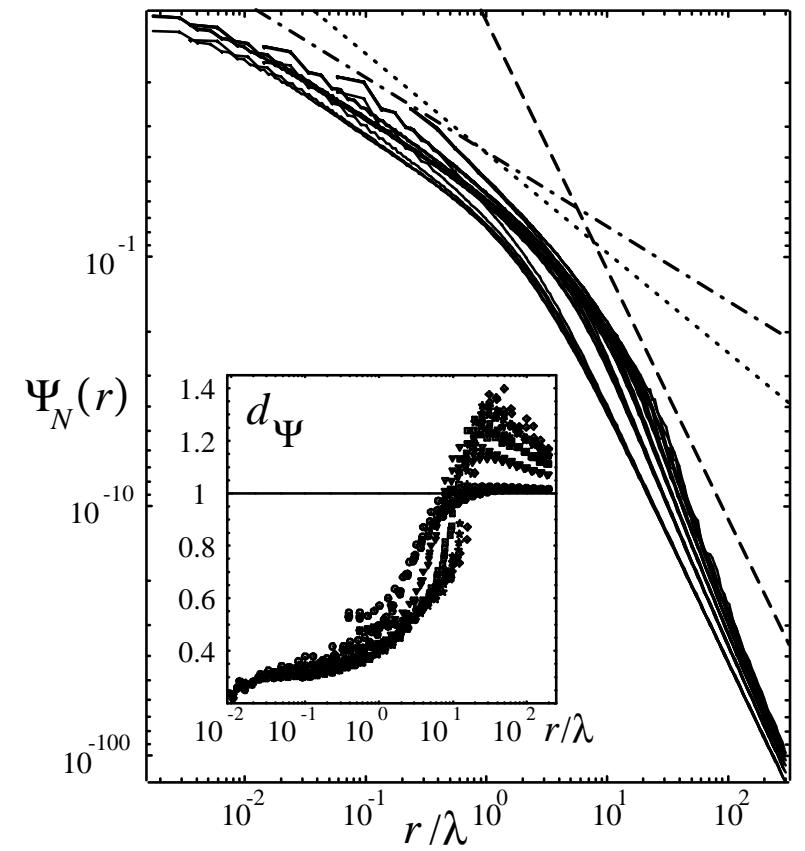

FIG. 7.: The decrease of the typical average amplitudes for $N$ configurations, $\Psi_{N}(r)$, versus $r / \lambda$ for electronic wave functions in the Anderson model in 1d for $N=1,16,256,4096$, and 65536 (from the bottom to the top) and five different strengths of disorder ( $w=0.35,0.5,1.0,2.0$, and 4.0). For the total number of configurations, the values of $\lambda(w)$, and the system sizes, see Fig. 2. The straight lines with the slopes $d_{\Psi}=1$ (dashed), $d_{\Psi}=0.4$ (dotted), and $d_{\Psi}=0.3$ (dot-dashed) are shown for comparison. In the insets, the effective local exponents $d_{\Psi}$ determined numerically from the slopes of the curves, are shown versus $r / \lambda$. The symbols correspond to the effective numbers of configurations $N=1$ (circles), 16 (triangles), 256 (squares), 4096 (stars), and 65536 (diamonds).

The special case $N=1$ in the definition corresponds to the "typical average" over one configuration. Since $\langle\psi(r)\rangle_{1} \equiv$ $\psi^{(\nu)}(r)$ in Eq. (21), the second step in the averaging procedure is dropped, and the values from each configuration are averaged logarithmically.

\section{B. Results in 1d}

Figure 6 shows $\Psi_{N}(r)$ for the electronic wave functions in $1 \mathrm{~d}$ for several $N$ values. The eigenfunctions are calculated for states in the band center $(E \approx 0)$ by the iteration method, 22 and the disorder parameter was chosen to be $w=1.0$. As can be seen in the Figure, the actual values of $\Psi_{N}(r)$ depend significantly on the number of configurations $N$ included in the averaging procedure. The local slopes of the curves yield the effective exponent $d_{\Psi}$ in our ansatz Eq. (15) which are shown in the inset of Fig. 6. One can see that the decay of $\Psi_{N}(r)$ is not simple exponential $\left(d_{\Psi}=1\right)$, but rather two different localization regimes with evidently different effective exponents $d_{\Psi}$ can be distinguished. 


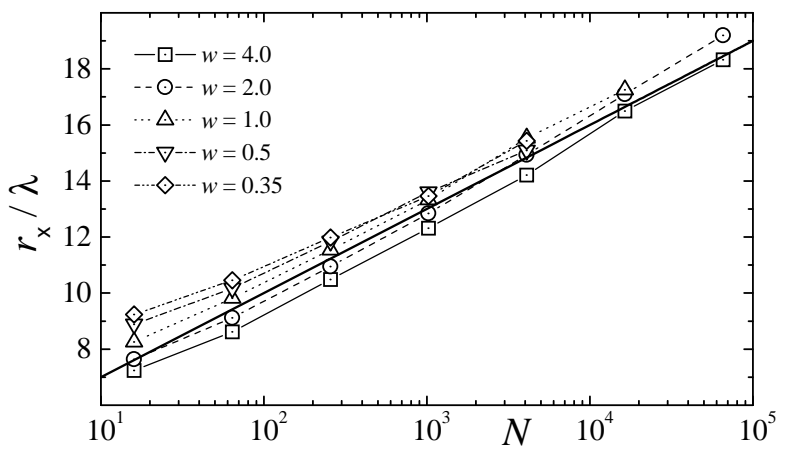

FIG. 8.: The crossover distances $r_{\times}(N) / \lambda$ for electronic wave functions in the Anderson model in $1 \mathrm{~d}$ are shown versus $N$ in a semi-logarithmic plot. The values of $r_{\times}(N)$ are determined as the lengths $r$, where the effective localization exponent $d_{\Psi}$ of $\Psi_{N}(r)$ intercepts with 1 . The symbols correspond to the different disorder strengths, $w=0.35$ (diamonds), 0.5 (stars), 1.0 (squares), 2.0 (triangles), and 4.0 (circles). The straight line $r_{\times}(N) / \lambda=$ $3 \log _{10} N+4$ is shown for comparison.

In the first localization regime, where $\Psi_{N}(r)$ decays roughly by one order of magnitude, we find a stretched exponential decay (sublocalization) with an effective exponent $d_{\Psi} \cong 0.4$ for all values of $N$, which is consistent with the result obtained in the previous Section. As expected, this sublocalization regime expands as the number of configurations $N$ increases.

In the second regime, for large $r$ values, self-averaging fails and $\Psi_{N}(r)$ depends explicitly on $N$. In the beginning of this regime, $d_{\Psi}$ is considerably larger than 1 , corresponding to a faster than exponential decay (superlocalization). Only for extremely large $r$ values the simple exponential decay $\left(d_{\Psi}=1\right)$ is reached. This behavior agrees nicely with the analytical results obtained in the previous Section, as can be seen by comparing the inset of Fig. 6 with the analytical values of the effective localization exponent $d_{\Psi}$ shown in Fig. 5. Note that very large $r$ values cannot be reached in the numerical calculations. Thus, the theoretical description, that becomes exact asymptotically for large $r$, is complementary to the numerics in this sense.

The localization behavior is similar for other strengths of disorder $w$. This can be seen in Fig. 7, where the data for five different $w$ has been included. Due to the scaling of the localization length, $\lambda=105 / w^{2}$, the data can be scaled onto master curves for each number of configurations $N$. For the second regime, where superlocalization occurs, we get an excellent data collapse. In the first regime, where sublocalization occurs, the data collapse is less accurate, because the localization exponent $d_{\Psi}$ depends on the disorder strength $w$. We obtain $d_{\Psi} \approx 0.3$ for small disorder $(w=0.5)$ and $d_{\Psi} \approx 0.4$ for larger disorder $(w=2.0)$.

The distance $r_{\times}$characterizing the crossover from the first $\left(r<r_{\times}\right)$to the second $\left(r>r_{\times}\right)$localization regime depends on the number of configurations $N$. Since $d_{\Psi}<1$ in the sublocalization regime (for $r<r_{\times}$) and $d_{\Psi}>1$ in the superlocalization regime (for $r>r_{\times}$), the values of $r_{\times}$ can be determined as the lengths $r$ where $d_{\Psi}$ intercepts with unity. Figure 8 shows $r_{\times}(N) / \lambda$ versus $\log N$. It is obvious that $r_{\times}(N)$ increases approximately logarithmically with $N$, $r_{\times}(N) / \lambda \approx 3 \log _{10} N+4$.

\section{Results in $2 d$}

Figure 9 shows our results for the mean amplitudes $\Psi_{N}(r)$ [defined in Eqs. (21) and (22)] for the Anderson model in $2 \mathrm{~d}$. We consider the same two disorder strengths and types of boundary conditions as in Figs. 3 and 4 and four values of $N$. The Figure shows clearly that at all distances from the localization center stretched exponentials (sublocalization) occur, which is fully consistent with the theoretical results obtained in Section IV (see especially Fig. 5). In addition, there is practically no dependence of the effective localization exponent, obtained from the local slopes in Fig. 9, on the number of configurations $N$ taken into account in the averaging procedure. The mean wave function amplitudes are just slightly shifted to larger values with increasing $N$.

In the small- $r$ regime, which cannot exactly be described by the theoretical study in Section IV, we find strong sublocalization characterized by $d_{\Psi} \approx 0.33$, similar to our results in $1 \mathrm{~d}$ where we also obtained values between 0.3 and 0.4 (see Figs. 7 and 8). This regime ranges over approximately two orders of magnitude in $r$ from the site neighboring the center of localization $(r=1)$ to $r_{\times}(N)$, which is $>100$ for large $N$ in the two examples considered. In this first regime the mean wave function amplitudes decay to values of $\approx 10^{-5}$. Thus, this regime is most relevant for most applications.

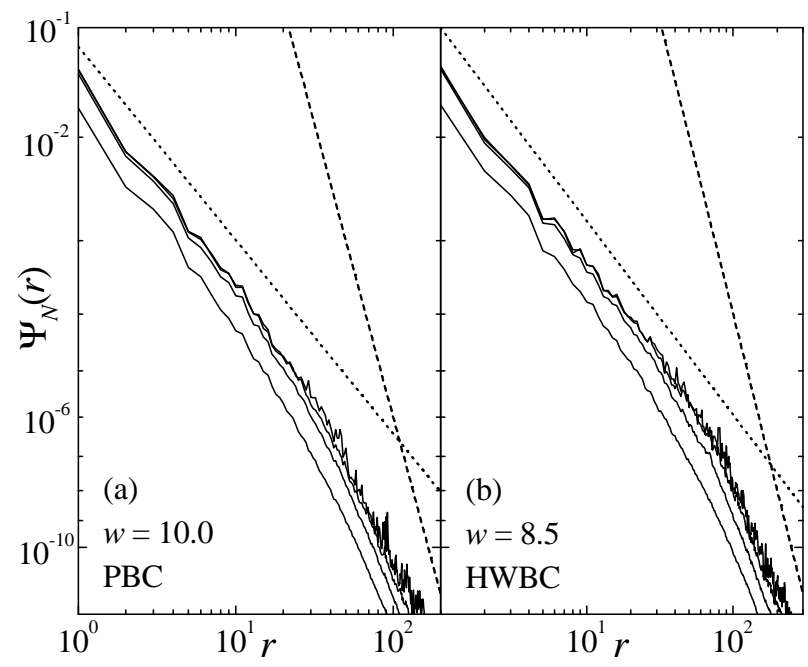

FIG. 9.: The decrease of $\Psi_{N}(r)$ versus $r$ for electronic wave functions in the Anderson model in $2 \mathrm{~d}$ for $N=1,8,64$, and 512 (from the bottom to the top). For (a) $w=8.5$ and HWBC were chosen, while $w=10.0$ and $\mathrm{PBC}$ in (b). For each part of the figure, $\approx 1000$ eigenfunctions with $E \approx 0$ were calculated using the Lanczos algorithm on a $300 \times 300$ lattice. The straight dashed and dotted lines have the slopes $d_{\Psi}=1$ and $d_{\Psi}=0.33$, respectively, and are shown for comparison. 
The second regime for large distances $r$ from the localization center $\left(r>r_{\times}\right)$can be seen only in a quite limited $r$ range, because of the limited system size and finite size effects especially for PBC. The effective localization exponents $d_{\Psi}$ in this regime are definitely larger than in the first regime, but we still obtain values remaining strictly below one. Thus, in $2 \mathrm{~d}$, the second regime is also characterized by sublocalization, in contrast to the superlocalization observed in 1d. This numerical result is in very nice agreement with the prediction from the theoretical description in Section IV.

\section{SUMMARY}

In order to discuss the localization and fluctuation behavior of localized eigenfunctions in the Anderson model we have studied the histogram amplitude distribution function $H(A, r)$ which corresponds to the probability density to find the amplitude $A \equiv-\ln |\psi|$ at distance $r$ from the localization center. We find that $H(A, r)$ quite exactly follows a log-normal shape for Anderson wave functions in $1 \mathrm{~d}$ and $2 \mathrm{~d}$. For increasing distance from the localization center, the log-normal fits become even better. Hence, the distributions can be characterized by two parameters, the localization length $\lambda$ and the fluctuation parameter $\sigma$ that is proportional to the width of the distributions. While both parameters exactly follow the predictions of the standard single parameter scaling theory in 1d, deviations occur in higher dimensions. The fluctuation parameter is smaller than the standard value of the single parameter scaling theory for the Anderson model in $2 \mathrm{~d}$, in contrast to a larger value for the percolation model. Furthermore, the localization length shows a logarithmic dependence on the distance from the localization center for the Anderson model in $2 \mathrm{~d}$, which gives an explanation for the contradicting values that have been published before and casts doubts on the existence of a finite asymptotic localization length.

Using the log-normal ansatz for the amplitude distributions, the decay of the average eigenfunctions can be calculated analytically. It is remarkable that by this simple approach, the essential features of the localization phenomenon, in 1d: sublocalization in the regime of small distances from the localization center, crossover to superlocalized behavior (that depends logarithmically on the number of configurations $N$ ), and final approach to simple exponential behavior, and in $2 \mathrm{~d}$ : sublocalization in all regimes, are reproduced.

\section{Acknowledgements}

We would like to thank Richard Berkovits for useful discussions and Lev Deych for helpful comments. Support from the Deutsche Forschungsgemeinschaft(DFG) and the German Academic Exchange Service (Deutscher Akademischer Austauschdienst, DAAD) is gratefully acknowledged.
${ }^{1}$ P. W. Anderson, Phys. Rev. 109, 1492 (1958).

${ }^{2}$ E. N. Economou, Green's functions in quantum physics, 2 nd ed. (Springer-Verlag, Berlin, 1990).

${ }^{3}$ B. Kramer and A. MacKinnon, Rep. Prog. Phys. 56, 1469 (1993).

${ }^{4}$ M. Janssen, Phys. Reports 295, 2 (1998).

${ }^{5}$ P. W. Anderson, D. J.Thouless, E. Abrahams, and D. S. Fisher, Phys. Rev. B 22, 3519 (1980).

${ }^{6}$ Fractals and Disordered Systems, edited by A. Bunde and S. Havlin (Springer-Verlag, Heidelberg, 1996), 2nd ed.; D. Stauffer and A. Aharony, Introduction to Percolation Theory, (Taylor \& Francis, London, 1992), 2nd ed.

${ }^{7}$ E. Abrahams, P. W. Anderson, D. C. Licciardello, and T. V. Ramakrishnan, Phys. Rev. Lett. 42, 673 (1979).

${ }^{8}$ L. I. Deych, A. A. Lisyansky, and B. L. Altshuler, Phys. Rev. Lett. 84, 2678 (2000); Phys. Rev. B 64, 224202 (2001).

${ }^{9}$ D. Braun, E. Hofstetter, G. Montambaux, and A. MacKinnon, Phys. Rev. B 64, 155107 (2001).

${ }^{10}$ M. Rühländer, P. Markos, and C. M. Soukoulis, Phys. Rev. B 64, 172202 (2001).

${ }^{11}$ K. Müller, B. Mehlig, F. Milde, and M. Schreiber, Phys. Rev. Lett. 78, 215 (1997).

${ }^{12}$ V. Uski, B. Mehlig, and R. A. Römer, Ann. Phys. 7, 437 (1998).

${ }^{13}$ L. I. Deych, D. Zaslavsky, and A. A. Lisyansky, Phys. Rev. Lett. 81, 5390 (1998)

${ }^{14}$ V. Uski, B. Mehlig, and M. Schreiber, Phys. Rev. B 63, 241101 (2001); V. Uski, B. Mehlig, R. A. Römer, and M. Schreiber, Physica B 284-288, 1934 (2001).

${ }^{15}$ B. K. Nikolic, Phys. Rev. B 64, 014203 (2001).

${ }^{16}$ E. Abrahams, S. V. Kravchenko, and M. P. Sarachik, Rev. Mod. Phys. 73, 251 (2001).

17 A. MacKinnon and B. Kramer, Phys. Rev. Lett. 47, 1546 (1981); Z. Phys. B 53, 1 (1983).

${ }^{18}$ M. Kappus and F. Wegner, Z. Phys. B 45, 15 (1981).

${ }^{19}$ J. L. Pichard, J. Phys. C 19, 1519 (1986).

${ }^{20}$ S. Russ, S. Havlin, and I. Webman, Philos. Mag. B 77, 1449 (1998).

${ }^{21}$ I. Kh. Zharekeshev, M. Batsch, and B. Kramer, Europhys. Lett. 34, 587 (1996).

${ }^{22}$ H.E. Roman and C. Wiecko, Z. Phys. B 62, 163 (1986).

${ }^{23}$ C. Lanczos, J. Res. Nat. Bur. Stand. 45, 255 (1950); J. Cullum and R. Willoughby, Lanczos algorithms for large symmetric eigenvalue computations, Vol. 1: Theory, 2: Programs, Progress in Scientific Computing, (Birkhäuser, Boston, 1985).

${ }^{24}$ M. L. Metha, Random Matrices (Academic Press, Boston, 1991); T. Guhr, A. Müller-Groeling, and H. A. Weidenmüller, Phys. Rep. 299, 190 (1998)

${ }^{25}$ B. I. Shklovskii, B. Shapiro, B. R. Sears, P. Lambrianides, and H. B. Shore, Phys. Rev. B 47, 11487 (1993).

${ }^{26}$ V. I. Fal'ko and K. B. Efetov, J. Math. Phys. 37, 4935 (1996).

${ }^{27}$ C. E. Porter and R. G. Thomas, Phys. Rev. 104, 483 (1956); T. A. Brody, J. Flores, J. B. French, P. A. Mello, A. Pandey, and S. S. M. Wong, Rev. Mod. Phys. 53, 385 (1981).

${ }^{28}$ J. W. Kantelhardt and A. Bunde, Phys. Rev. E 56, 6693 (1997).

${ }^{29}$ S. Kirkpatrick and T. P. Eggarter, Phys. Rev. B 6, 3598 (1972).

${ }^{30}$ C. M. Soukoulis, Q. Li, and G. S. Grest, Phys. Rev. B 45, 7724 (1992); I. Chang, Z. Lev, A. B. Harris, J. Adler, and A. Aharony, Phys. Rev. Lett. 74, 2094 (1995); R. Berkovits and Y. Avishai, 
Phys. Rev. B 53, R16125 (1996); A. Kaneko and T. Ohtsuki, J. Phys. Soc. Jqn. 68, 1488 (1999).

${ }^{31}$ A. Bunde and J. Dräger, Phys. Rev. E 52, 53 (1995); J. Dräger and A. Bunde, ibid 54, 4596 (1996).
${ }^{32}$ A. Bunde, J. Dräger, and J. W. Kantelhardt, in: Anomalous Diffusion: From Basics to Applications, Lecture Notes in Physics, edited by A. Pekalski and K. Sznajd-Weron, p. 262 (Springer, Berlin, 1999). 